Vol. $69(2004) \quad[107-111]$

\title{
ON BIORTHOGONAL SYSTEMS AND MAZUR'S INTERSECTION PROPERTY
}

\author{
JAN RYCHTÁr̆
}

We give a characterisation of Banach spaces $X$ containing a subspace with a shrinking Markushevich basis $\left\{x_{\gamma}, f_{\gamma}\right\}_{\gamma \in \Gamma}$. This gives a sufficient condition for $X$ to have a renorming with Mazur's intersection property.

A biorthogonal system in a Banach space $X$ is a subset $\left\{x_{\gamma}, f_{\gamma}\right\}_{\gamma \in \Gamma} \subset X \times X^{*}$ such that $f_{\gamma}\left(x_{\gamma^{\prime}}\right)=\delta_{\gamma \gamma^{\prime}}$ for $\gamma, \gamma^{\prime} \in \Gamma$. The biorthogonal system $\left\{x_{\gamma}, f_{\gamma}\right\}_{\gamma \in \Gamma}$ in $X$ is called fundamental if $X=\overline{\operatorname{span}}\left\{x_{\gamma} ; \gamma \in \Gamma\right\}$. A Markushevich basis is a fundamental biorthogonal system $\left\{x_{\gamma}, f_{\gamma}\right\}_{\gamma \in \Gamma}$ in $X$ such that $\left\{f_{\gamma}\right\}_{\gamma \in \Gamma}$ separates points of $X$. A Markushevich basis $\left\{x_{\gamma}, f_{\gamma}\right\}_{\gamma \in \Gamma} \subset X \times X^{*}$ is called shrinking if $X^{*}=\overline{\operatorname{span}}\left\{f_{\gamma} ; \gamma \in \Gamma\right\}$. In this note we use $\Gamma$ as a cardinal number.

A Banach space $X$ is said to be an Asplund space, if every separable subspace of $X$ has a separable dual. A Banach space $X$ has Mazur's intersection property if every bounded closed convex set can be represented as an intersection of closed balls. A density of a topological space is the least cardinality of a dense set. We refer to [2] for undefined terms used in this paper.

It is known, [9, Theorem 7.18, Theorem 7.12], that if a dual unit ball of a Banach space $X$ is a Corson compact, then dens $X=w^{*}$-dens $X^{*}$ and the following are equivalent.

(i) $X$ has a shrinking Markushevich basis,

(ii) $X$ is an Asplund space,

(iii) $X$ admits a Fréchet smooth norm.

Let us remark that if a norm on $X$ is Fréchet smooth, then $X$ has Mazur's intersection property, [1, Proposition 4.5].

When we do not assume that the dual unit ball is a Corson compact, then the above is no longer true. For example, the Banach space $C(K)$, where $K$ is a Kunen's compact $($ see $[8,5])$, is an Asplund space without shrinking Markushevich basis and without Mazur's intersection property $([6])$.

Received 15th July, 2003

Research supported by NSERC 7926, FS Chia Ph.D. Scholarship for 2002/2003 and GAUK 277/2001, written as part of Ph.D. thesis under supervision of Professor N. Tomczak-Jaegermann and Professor V. Zizler. The author wishes to thank Professor G. Godefroy and Professor S. Todorčević for discussions on the subject of this note.

Copyright Clearance Centre, Inc. Serial-fee code: 0004-9727/04 \$A2.00+0.00. 
The aim of this note is to prove a theorem in the spirit of equivalences above but without assuming anything about a dual unit ball.

THEOREM 1. Let $E$ be a Banach space. Then the following are equivalent.

(i) There is a subspace $Y \subset E$ with a shrinking Markushevich basis $\left\{x_{\gamma}, f_{\gamma}\right\}_{\gamma \in \Gamma}$.

(ii) There is an Asplund space $X \subset E$ with dens $X=w^{*}$-dens $X^{*}=\Gamma$.

(iii) There is a subspace $Z \subset E$ that admits a Fréchet smooth norm and such that dens $Z=w^{*}$-dens $Z^{*}=\Gamma$.

Moreover, if one from the above occurs with $\Gamma=\operatorname{dens} E^{*}$, then

(iv) $E$ admits a norm with the Mazur intersection property.

REMARK. The condition dens $E=\operatorname{dens} E^{*}$ is necessarily for renorming with Mazur intersection property due to $[3]$.

Proof: Implications (i) $\Rightarrow$ (iii) $\Rightarrow$ (ii). If $Y$ has a shrinking Markushevich basis, then $Y$ admits a Fréchet differentiable norm [2, Theorem 11.23]. Thus it is an Asplund space [2, Theorem 8.24]. It remains to show that $w^{*}$-dens $Y^{*}=\operatorname{dens} Y=\Gamma$. Let $\left\{g_{\alpha} ; \alpha \in A\right\} \subset Y^{*}$ be a weak $^{*}$ dense set. As the basis $\left\{x_{\gamma}, f_{\gamma}\right\}_{\gamma \in \Gamma}$ is shrinking, we may assume without loss of generality that $\left\{g_{\alpha} ; \alpha \in A\right\} \subset \operatorname{span}\left\{f_{\gamma} ; \gamma \in \Gamma\right\}$. For a contradiction, assume that $|A|<\Gamma$. Thus there is $\Gamma^{\prime}<\Gamma$ such that

$$
\left\{g_{\alpha} ; \alpha \in A\right\} \subset \operatorname{span}\left\{f_{\gamma} ; \gamma \in \Gamma^{\prime}\right\} .
$$

Hence, for $\gamma \in \Gamma \backslash \Gamma^{\prime}$ and all $\alpha \in A$

$$
\left|\left(f_{\gamma}-g_{\alpha}\right)\left(x_{\gamma}\right)\right|=1
$$

a contradiction with the density of $\left\{g_{\alpha} ; \alpha \in A\right\}$.

Implication (i) $\Rightarrow$ (iv). Due to [6, Theorem 2.4], to show that $E$ admits a norm with the Mazur intersection property, it is enough to construct a fundamental biorthogonal system $\left\{q_{\gamma}, x_{\gamma}\right\}_{\gamma \in \Gamma} \subset E^{*} \times E$. As we assume that $Y \subset E$ has a shrinking Markushevich basis, that is a fundamental biorthogonal system $\left\{f_{\gamma}, x_{\gamma}\right\}_{\gamma \in \Gamma} \subset Y^{*} \times Y$, we only need to show the following.

Lemma 2. Let $E$ be a Banach space with dens $E^{*}=\Gamma$ and $Y \subset E$ be a closed subspace. Assume that there is a fundamental biorthogonal system $\left\{f_{\gamma}, x_{\gamma}\right\}_{\gamma \in \Gamma} \subset Y^{*} \times Y$. Then there is a fundamental biorthogonal system $\left\{q_{\gamma}, x_{\gamma}\right\}_{\gamma \in \Gamma} \subset E^{*} \times E$.

Proof: By a relabeling and rescaling, we may have a fundamental system $\left\{f_{\gamma}^{n}, x_{\gamma}^{n}\right\}_{\gamma \in \Gamma, n \in \mathbb{N}} \subset Y^{*} \times Y$ such that for every $\gamma \in \Gamma, \lim _{n}\left\|f_{\gamma}^{n}\right\|=0$. By the Hahn-Banach theorem, consider $f_{\gamma}^{n} \in E^{*}$. Let $\left\{g_{\gamma}\right\}_{\gamma \in \Gamma}$ be a dense set of $B_{E^{*}} \cap Y^{\perp}$.

We claim, that $A=\left\{g_{\gamma}+f_{\gamma}^{n}\right\}_{\gamma \in \Gamma, n \in \mathbb{N}}$ is linearly dense in $E^{*}$. Indeed, let $G \in E^{* *}$ be such that $G(f)=0$ for every $f \in A$. Then $G\left(g_{\gamma}\right)=\lim _{n} G\left(g_{\gamma}+f_{\gamma}^{n}\right)=0$ and thus $G \in\left(Y^{\perp}\right)^{\perp}=Y^{* *}$. Hence $G=0$ as $\left\{f_{\gamma}^{n}\right\}_{\gamma \in \Gamma, n \in \mathbb{N}}$ are linearly dense in $Y^{*}$. 
Hence $\left\{g_{\gamma}+f_{\gamma}^{n}, x_{\gamma}^{n}\right\}_{\gamma \in \Gamma, n \in \mathbb{N}} \subset E^{*} \times E$ is a fundamental biorthogonal system.

REMARK. As $c_{0}(\Gamma) \subset C([0, \Gamma])$, Lemma 2 provides a direct proof of the fact that there is a fundamental biorthogonal system $\left\{f_{\gamma}, x_{\gamma}\right\}_{\gamma \in \Gamma} \subset C([0, \Gamma])^{*} \times C([0, \Gamma])$. Thus $C([0, \Gamma])$ admits a norm with Mazur's intersection property, see also [6, Lemma 3.5].

Thus it remains to prove the implication (ii) $\Rightarrow$ (i).

The proof goes in the spirit of [7, Theorem 1.a.5] and [4]. We shall use the concept of the Jayne-Rogers selector, see [1, Chapter 1]. The Jayne-Rogers selection map $\mathcal{D}^{X}$ on an Asplund space $X$ is a multi-valued map that satisfies the following.

(i) $\mathcal{D}^{X}(x)=\left\{D_{n}^{X}(x) ; n \in \mathbb{N}\right\} \cup D_{\infty}^{X}(x) \subset X^{*}$,

(ii) $D_{n}^{X}$, for $n \in \mathbb{N}$, are continuous functions from $X$ to $X^{*}$,

(iii) $D_{\infty}^{X}(x)=\lim _{n \rightarrow \infty} D_{n}^{X}(x)$ for every $x \in X$,

(iv) $D_{\infty}^{X}(x)(x)=\|x\|^{2}=\left\|D_{\infty}^{X}(x)\right\|^{2}$,

(v) $X^{*}=\overline{\operatorname{span}} \mathcal{D}^{X}(X)$.

Such selector exists by [1, Theorem 1.5.2].

In order to construct $Y \subset X$ we shall define, by a transfinite induction, vectors $x_{\alpha+1} \in X$, subspaces $Y_{\alpha} \subset X$ and subsets $F_{\alpha} \subset X^{*}$, for all $\alpha<\Gamma$. Put $Y_{0}=0$ and $F_{0}=0$ and pick arbitrary nonzero $x_{1} \in\left(F_{0}\right)_{\perp}=\left\{x \in X ; f(x)=0\right.$ for all $\left.f \in F_{0}\right\}$. Then put $Y_{1}=\operatorname{span}\left\{x_{1}\right\}$, and $F_{1}=\left\{\mathcal{D}^{X}(x) ; x \in Y_{1}\right\}$. Let $Y_{\alpha}$ and $F_{\alpha}$ for $\alpha<\Gamma$ have been chosen. Notice that dens $Y_{\alpha}<\Gamma$ and thus dens $F_{\alpha} \leqslant \aleph_{0}$.dens $Y_{\alpha}<\Gamma$. Thus $F_{\alpha}$ is not $w^{*}$-dense and we can pick a nonzero vector $x_{\alpha+1} \in\left(F_{\alpha}\right)_{\perp}$. Set $Y_{\alpha+1}=\operatorname{span}\left\{Y_{\alpha} \cup\left\{x_{\alpha+1}\right\}\right\}$ and $F_{\alpha+1}=\left\{\mathcal{D}^{X}(x) ; x \in Y_{\alpha+1}\right\}$.

If $\alpha \leqslant \Gamma$ is a limit ordinal, define $Y_{\alpha}=\overline{\operatorname{span}} \cup_{\beta<\alpha} Y_{\beta}$ and $F_{\alpha}=\left\{\mathcal{D}^{X}(x), x \in Y_{\alpha}\right\}$.

Put $Y=\overline{\text { span }} \cup_{\alpha<\Gamma} Y_{\alpha}$. We shall show that $Y$ has a shrinking Markushevich basis $\left\{x_{\alpha+1}, f_{\alpha+1}\right\}_{\alpha<\Gamma}$, where $\left\{x_{\alpha+1}\right\}_{\alpha<\Gamma}$ have been already chosen and their biorthogonals $f_{\alpha+1}$ will be defined by projections.

Clearly $Y=\overline{\operatorname{span}}\left\{x_{\alpha+1} ; a<\Gamma\right\}$. Let us define projections $P_{\alpha}: Y \rightarrow Y_{\alpha}$ for all $\alpha \leqslant \Gamma$. First define projections $\widetilde{P}_{\alpha}: \operatorname{span}\left\{x_{\alpha+1} ; \alpha<\Gamma\right\} \rightarrow Y_{\alpha}$ by letting $P_{\alpha}\left(x_{\beta}\right)=x_{\beta}$ if $\beta \leqslant \alpha$ and 0 otherwise. $\widetilde{P}_{\alpha}$ are well defined and once we show that they all have norm 1 , they will extend naturally onto desired projections on $Y$.

Take $x \in \operatorname{span}\left\{x_{\alpha+1} ; \alpha<\Gamma\right\}$ and fix $\alpha \leqslant \Gamma$. Then by the properties of the JayneRogers selector and due to the choice of $\left\{x_{\alpha+1} ; \alpha<\Gamma\right\}$ we have

$$
\begin{aligned}
\left\|\widetilde{P}_{\alpha}(x)\right\|^{2} & =D_{\infty}^{X}\left(\widetilde{P}_{\alpha}(x)\right)\left(\widetilde{P}_{\alpha}(x)\right)=D_{\infty}^{X}\left(\widetilde{P}_{\alpha}(x)\right)(x) \\
& \leqslant\|x\| .\left\|D_{\infty}^{X}\left(\widetilde{P}_{\alpha}(x)\right)\right\|=\|x\| .\left\|\widetilde{P}_{\alpha}(x)\right\| .
\end{aligned}
$$

Thus $\left\|\widetilde{P}_{\alpha}\right\|=1$.

Define $f_{\alpha+1} \in Y^{*}$ for $\alpha<\Gamma$ such that $\left\|f_{\alpha+1}\right\|=1$ and $f_{\alpha+1} \in\left(P_{\alpha+1}-P_{\alpha}\right)^{*} Y^{*}$. Clearly $\left\{x_{\alpha+1}, f_{\alpha+1}\right\}_{\alpha<\Gamma}$ is a biorthogonal system. 
We shall show that the projection $\left\{P_{\alpha}\right\}_{\alpha<\Gamma}$ are shrinking. From that it follows that $\overline{\operatorname{span}}\left\{f_{\alpha+1} ; \alpha<\Gamma\right\}=Y^{*}$.

Let $\alpha \leqslant \Gamma$ be a fixed limit ordinal and set $Z=P_{\alpha} Y$. Let $f \in Z^{*}$ be arbitrary. We need to show that there exist a sequence of ordinals $\beta_{n} \rightarrow \alpha$ and $g_{n} \in P_{\beta_{n}}^{*} Z^{*}$ such that $g_{n} \rightarrow f$ in $Z^{*}$. Fix $\varepsilon>0$. Denote $\mathcal{D}^{Z}$ the restriction of $\mathcal{D}^{X}$ on $Z$, that is $D_{k}^{Z}(z)=\left.D_{k}^{X}(z)\right|_{Z}$ for all $z \in Z$. Clearly $\mathcal{D}^{Z}$ is the Jayne-Rogers selection map for $Z$. As $Z \subset X$ is an Asplund space, $Z^{*}=\overline{\operatorname{span}} \mathcal{D}^{Z}(Z)$. Thus

$$
\left\|f-\left(\sum_{i=1}^{n} D_{k_{i}}^{Z}\left(z_{i}\right)+\sum_{i=n+1}^{m} D_{\infty}^{Z}\left(z_{i}\right)\right)\right\|<\varepsilon
$$

where $k_{i} \in \mathbb{N}$, for $i=1, \ldots, n$ and $z_{i} \in Z$, for $i=1, \ldots, m$. Because $D_{\infty}^{Z}$ is a pointwise limit of $D_{n}^{Z}$, there are $k_{i} \in \mathbb{N}, i=n+1, \ldots, m$ such that

$$
\left\|f-\sum_{i=1}^{m} D_{k_{i}}^{Z}\left(z_{i}\right)\right\|<\varepsilon
$$

Because $D_{n}^{Z}$ are continuous, there is $\beta<\alpha$ such that

$$
\left\|f-\sum_{i=1}^{m} D_{k_{i}}^{Z}\left(z_{i}^{\prime}\right)\right\|<\varepsilon
$$

for $z_{i}^{\prime} \in P_{\beta} Z$.

Thus it remains to show that $\mathcal{D}^{Z}\left(P_{\beta}(Z)\right) \subset P_{\beta}^{*} Z^{*}$ for $\beta<\alpha$. Let $z \in P_{\beta} Z$. By the choice of $\left\{x_{\alpha+1} ; \alpha<\Gamma\right\}$ we know that $\mathcal{D}^{Z}(z)\left(x_{\gamma}\right)=0$ for $\gamma>\beta$. Thus

$$
P_{\beta}^{*}\left(\mathcal{D}^{Z}(z)\right)(x)=\mathcal{D}^{Z}(z)\left(P_{\beta} x\right)=\mathcal{D}^{Z}(z)(x),
$$

for all $x \in Z$, and it was exactly what we needed to prove.

\section{REFERENCES}

[1] R. Deville, G. Godefroy and V. Zizler, Smoothness and renormings in Banach spaces, Pitmen Monographs and Surveys in Pure and Applied Mathematics 64 (Longman Scientific and Technical, Harlow, 1993).

[2] M. Fabian, P. Habala, P. Hájek, V. Montesinos, J. Pelant and V. Zizler, Functional analysis and infinite dimensional geometry, CMS Books in Mathematics 8 (Springer-Verlag, New York, 2001).

[3] J.R. Giles, D.A. Gregory, and B. Sims, 'Characterization of normed linear spaces with Mazur's intersection property', Bull. Austral. Math. Soc. 18 (1978), 471-476.

[4] G. Godefroy, 'Asplund spaces and decomposable nonseparable Banach spaces', Rocky Mountain J. Math. 25 (1995), 1013-1024.

[5] P. Holický, M. Šmídek, L. Zajićek, 'Convex functions with non-Borel set of Gâteaux differentiability points', Comment. Math. Univ. Carolin. 39 (1998), 469-482. 
[6] M. Jiménez Sevilla and J. P. Moreno, 'Renorming Banach spaces with the Mazur intersection property', J. Funct. Anal. 144 (1997), 486-504.

[7] J. Lindernstrauss and L. Tzafriri, Classical Banach spaces I (Springer-Verlag, Berlin, Heidelberg, New York 1977).

[8] S. Negrepontis, 'Banach spaces and topology', in Handbook of Set Theoretic Topology, (K. Kunen and J.E. Vaughan, Editors) (North-Holland, Amsterdam, 1984), pp. 1045-1142.

[9] V. Zizler, 'Nonseparable Banach spaces', in Handbook of the geometry of Banach spaces, Vol. II, (W.B. Johnson and J. Lindenstrauss, Editors). (Elsevier, Amsterdam, 2003).

Department of Mathematical and Statistical Science

University of Alberta

Edmonton, Alberta T6G 2G1

Canada

e-mail: jrychtar@math.ualberta.ca 